\title{
Sorovares de Listeria monocytogenes e espécies relacionadas, isoladas de material clínico humano
}

\author{
Serovars of Listeria monocytogenes and related species \\ isolated from human clinical specimens
}

\author{
Ernesto Hofer ${ }^{1}$, Cristhiane Moura Falavina dos Reis ${ }^{1}$ e Cristina Barroso Hofer ${ }^{2}$
}

\begin{abstract}
RESUMO
A análise fenotípica de 255 amostras do gênero Listeria isoladas de material clínico humano, tanto de indivíduos doentes (220-86,3\%), como de aparentemente normais (35-13,7\%) de várias regiões do país e colecionadas no período de 1969 a 2000, permitiu caracterizar a distribuição de sorovares de Listeria monocytogenes. Nas faixas etárias de 0 a 10 e de 41 a 60 anos, predominaram os isolamentos de líquido cefalorraquidiano sobre os de sangue, incluindo dos transplantados renais. Somente dos hemocultivos foi possível detectar os sete sorovares de Listeria monocytogenes. No cômputo geral, o sorovar $4 \mathrm{~b}$ foi o mais incidente (154-60,3\%) secundado por $1 / 2$ a (74-29\%) nos três decênios considerados, além de ocorrerem em quase todas as regiões do país. Os dados deste estudo evidenciaram a circulação de L. monocytogenes na espécie humana, provocando quadros graves de meningite e septicemia, bem como, revelando a figura do portador assintomático, razão pela qual são recomendadas novas investigações bacteriológicas, subsidiadas por análises clínico-patológicas e epidemiológicas.
\end{abstract}

Palavras-chaves: Listeria. Espécies. Sorovares. Listeriose humana.

\begin{abstract}
Using phenotype techniques, characterization was realized of species and serovars of 255 strains of Listeria isolated from human material: $220-86.3 \%$ were from patients with possible invasive disease, while 35-13.7\% were from colonized healthy individuals. The strains were collected in several regions of Brazil from 1969-2000. In individuals aged 0-10 or 41-60 years old Listeria monocytogenes was isolated more often in cerebral spinal fluid than in blood cultures, including samples from renal transplant recipients. All Listeria monocytogenes serovars were detected in blood culture strains. The predominant serovars characterized were $4 \mathrm{~b}$ (154-60.3\%) and $1 / 2$ a (74-29\%). In this study, Listeria monocytogenes causing invasive diseases, such as meningitis or septicemia, or colonizing individuals, were identified. Consequently further studies focusing on clinical and pathological as well as epidemiological issues, including risk factors associated with foodborne transmission should be pursued.
\end{abstract}

Key-words: Listeria. Species. Serovars. Human listeriosis.

A listeriose humana tem como agente etiológico a Listeria monocytogenes um bastonete Gram-positivo, anaeróbio facultativo e não esporulado. 0 microrganismo está amplamente disseminado em natureza, envolvendo um elevado número de fontes de infecção, incluindo o homem e utilizando vários mecanismos de transmissão $0^{6}$.

A partir da década de 80 do século passado, foi definitivamente demonstrada a veiculação da bactéria através de inúmeros alimentos, inclusive resultando em alguns surtos de grande repercussãa ${ }^{25}$. É provável que a maioria dos casos humanos esporádicos tenham como mecanismo de transmissão da L. monocytogenes, os alimentos.

Diante das razões expostas, depreende-se que um dos pontos fundamentais no estudo da listeriose humana, reside no seu diagnóstico laboratorial, desde 0 isolamento até as definições das espécies e sorovares do gênero Listeria.

\footnotetext{
1. Laboratório de Zoonoses Bacterianas do Departamento de Bacteriologia do Instituto Oswaldo Cruz da Fundação Oswaldo Cruz, Rio de Janeiro, RJ. 2. Serviço de Doenças Infecciosas e Parasitárias do Hospital Universitário Clementino Fraga Filho da Universidade Federal do Rio de Janeiro, Rio de Janeiro, RJ. Órgão Financiador: Projeto parcialmente financiado pelo CNPq n ${ }^{\circ} 500415 / 2003-0$.

Endereço para correspondência: Dr. Ernesto Hofer. Depto ${ }^{\circ}$ de Bacteriologia/IOC/FIOCRUZ. Av. Brasil 4365, Manguinhos, $21040-900$ Rio de Janeiro, RJ. Tel: 5521 2598-4277 Ramal 332; Fax: 5521 2270-6565 Ramal 330

e-mail: hofer@uninet.com.br

Recebido para publicação em 3/12/2004

Aceito em 30/9/2005
} 
Tendo em vista as discretas referências sobre este aspecto em nosso meio ${ }^{11} 12$ procurou-se nesta investigação abordar retrospectivamente, as ocorrências de espécies e sorovares isolados de espécimes clínicos, nos três últimos decênios.

\section{MATERIAL E MÉTODOS}

Foram analisadas 255 amostras de Listeria, isoladas de diferentes materiais clínicos pelo próprio laboratório, assim como de outras instituições do país, durante o período compreendido entre 1969, 1972 a 2000 (Tabela 1). Todas as cepas estavam mantidas à temperatura de $4-8^{\circ} \mathrm{C}$ em tubos contendo meio semi-sólido em camada alta (Caldo TryptoseDifco ou similar acrescido de $0,4 \mathrm{~g} \%$ de agar-Difco ou similar), vedados com rolhas de borracha esterilizadas.

A identificação fenotípica foi realizada com base nas características morfotintoriais (método de Gram), associada ao perfil bioquímico de acordo com Rocourt cols ${ }^{29}$, incluindo-se rotineiramente a pesquisa da catalase e o comportamento no teste de CAMP com os indicadores - Staphylococcus aureus e Rhodococcus equi.

Na caracterização antigênica dos sorogrupos e sorovares, adotou-se a orientação de Seeliger \& Höhne ${ }^{33}$, recorrendo aos anti-soros somáticos e flagelares policlonais polivalentes e monovalentes produzidos pelo Laboratório de Zoonoses Bacterianas, Instituto Oswaldo Cruz/FIOCRUZ, RJ.

As análises estatísticas foram realizadas através do programa EPI-INFO 2000 (CDC). Na análise das variáveis categóricas recorreu-se ao teste exato de Fisher, bicaudal, sendo utilizado nas variáveis contínuas o teste de Mann-Whitney. Diferenças com 0 valor de $p \leq 0,05$ foram consideradas significantes.

\section{RESULTADOS}

No período em questão, foram analisadas 266 culturas com um diagnóstico presuntivo e/ou conclusivo de Listeria. Destas, 255 confirmaram após as análises fenotípicas como pertencentes ao gênero e as espécies, representando 95,8\% de acertos. As onze cepas excluídas originaram-se de fezes (5), secreção vaginal (4), líquido cefalorraquidiano (1) e sangue (1), nas quais predominaram diplococos Grampositivos, imóveis e catalase negativos (8) e bastonetes Gramnegativos fermentadores (3).

Os dados demográficos obtidos resultaram das anotações que acompanhavam as amostras, referindo principalmente a idade, como pode ser observado na Tabela 1 , onde foram relacionados os grupos etários. Já nas 165 amostras, em que estavam consignados os sexos dos doentes e portadores, predominaram os indivíduos do sexo masculino (95-57,5\%) sobre as pacientes (70-42,5\%), mas sem significância. Em ambos os parâmetros, assinala-se o problema da precariedade das informações, tendo em vista que $77(30,1 \%)$ pessoas na idade e $90(35,3 \%)$ quanto ao sexo, foram rotuladas como sem identificação.

Como detalhe de importância, verifica-se na Tabela 1 a discreta predominância de L. monocytogenes nas faixas etárias extremas (0-10 anos: 20,4\% e 44-60 anos: 28,7\%), embora não apresentando diferenças significativas.

Quanto à origem geográfica em relação as fontes de isolamento, destaca-se que as amostras oriundas dos Estados de São Paulo e Rio de Janeiro representaram 78,7\% (201) da amostragem analisada, bem como, é evidente a nítida predominância das cepas de $L$. monocytogenes oriundas de líquido cefalorraquidiano e de sangue, totalizando 217 (86,1\%) como mostra a Tabela 2.

Associando-se as distribuições das espécies e sorovares com suas origens geográficas (Tabela 3), salienta-se a predominância do sorovar $4 \mathrm{~b}$ (154-60,3\%), ocorrendo em todas as regiões, exceto na Bahia e tendo em segundo plano $\frac{1}{2}$ a com 74 (29\%) amostras distribuídos na maioria dos estados. Os demais sorotipos de $L$. monocytogenes apresentaram uma frequiência discreta (17-6,7\%). Dentre os nove estados que encaminharam as amostras, o sorovar $4 \mathrm{~b}$ predominou em cinco regiões, enquanto o sorotipo $1 \frac{1}{2}$ a sobressaiu no Paraná e Bahia.

De forma similar, o confronto dos sorovares de L. monocytogenes com as fontes de isolamento (Tabela 4), evidencia a nítida predominância do tipo 4b nos líquidos cefalorraquidianos, enquanto nos isolamentos de hemocultivos,

Tabela 1 - Relação de grupos etários com as fontes de isolamento de membros do gênero Listeria isolados no período de 1969-1972-2000.

\begin{tabular}{|c|c|c|c|c|c|c|c|c|c|c|}
\hline \multirow{2}{*}{$\begin{array}{l}\text { Grupos etários } \\
\text { (anos) }\end{array}$} & \multicolumn{8}{|c|}{ Fontes de isolamento } & \multicolumn{2}{|c|}{ Total } \\
\hline & LCR $^{*}$ & sangue & sangue $^{* * *}$ & fezes & secreção vaginal & adenite cervical & fígado (biópsia) & lesão cutânea & $\mathrm{n}^{0}$ & $\%$ \\
\hline $0-1$ & 18 & 10 & & & & & & & 28 & 11,0 \\
\hline $2-10$ & 13 & 8 & & 2 & & & 1 & & 24 & 9,4 \\
\hline $11-20$ & 5 & 1 & & 1 & & & & & 7 & 2,7 \\
\hline $21-30$ & 8 & 3 & & 3 & 4 & & & & 18 & 7,1 \\
\hline $31-40$ & 8 & 4 & 5 & 8 & 3 & & & & 28 & 11,0 \\
\hline $41-50$ & 15 & 7 & 4 & 10 & & & & 1 & 37 & 14,5 \\
\hline $51-60$ & 20 & 10 & 1 & 4 & & 1 & & & 36 & 14,2 \\
\hline S/identificação & 53 & 24 & & & & & & & 77 & 30,1 \\
\hline \multirow{2}{*}{$\begin{array}{cc}\text { Total } & \mathrm{n}^{0} \\
& \%\end{array}$} & 140 & 67 & 10 & 28 & 7 & 1 & 1 & 1 & 255 & 100,0 \\
\hline & 54,9 & 26,2 & 11,0 & 4 & 2,7 & 0,4 & 0,4 & 0,4 & & \\
\hline
\end{tabular}

*Líquido cefalorraquidiano.**Transplantados renais. 
Tabela 2 - Freqüência de membros do gênero Listeria em espécimes clínicos e distribuídos segundo a origem geográfica.

\begin{tabular}{|c|c|c|c|c|c|c|c|c|c|c|c|}
\hline \multirow[t]{2}{*}{ Fontes de isolamento } & \multicolumn{9}{|c|}{ Unidades Federadas* } & \multicolumn{2}{|c|}{ Total } \\
\hline & PE & BA & GO & DF & MG & $\mathrm{RJ}$ & SP & PR & RS & $\mathrm{n}^{0}$ & $\%$ \\
\hline LCR & 7 & & & 3 & 3 & 31 & 86 & 8 & 2 & 140 & 54,9 \\
\hline Sangue & 6 & 2 & & 1 & & 24 & 24 & 8 & 2 & 67 & 26,2 \\
\hline Sangue (transplantados) & & & & & & 3 & 7 & & & 10 & 4,0 \\
\hline Fezes & & & 10 & & & 15 & 3 & & & 28 & 11,0 \\
\hline Secreção vaginal & 2 & & & & & 5 & & & & 7 & 2,7 \\
\hline Adenite & & & & & & 1 & & & & 1 & 0,4 \\
\hline Fígado & & & & & & 1 & & & & 1 & 0,4 \\
\hline Lesão cutânea & & & & & & 1 & & & & 1 & 0,4 \\
\hline Total $\mathrm{n}^{\underline{0}}$ & 15 & 2 & 10 & 4 & 3 & 81 & 120 & 16 & 4 & 255 & 100,0 \\
\hline$\%$ & 5,9 & 0,8 & 4,0 & 1,6 & 1,2 & 31,7 & 47,0 & 6,2 & 1,6 & & \\
\hline
\end{tabular}

*PE: Pernambuco; BA: Bahia; GO: Goiás; DF: Distrito Federal; MG: Minas Gerais; RJ: Rio de Janeiro; SP: São Paulo PR: Paraná; RS: Rio Grande do Sul.

Tabela 3 - Distribuição das espécies e sorovares de Listeria isolados de material clínico humano segundo a origem geográfica (estados).

\begin{tabular}{|c|c|c|c|c|c|c|c|c|c|c|c|c|c|}
\hline \multirow[t]{2}{*}{ Estados } & \multicolumn{7}{|c|}{ Listeria monocytogenes } & \multicolumn{3}{|c|}{ Listeria innocua } & \multirow[b]{2}{*}{ Listeria grayi } & \multicolumn{2}{|c|}{ Total } \\
\hline & $1 / 2 \mathrm{a}$ & $1 / 2 \mathrm{~b}$ & $1 / 2 \mathrm{C}$ & $3 a$ & $4 a$ & $4 a b$ & $4 \mathrm{~b}$ & $6 a$ & $6 \mathrm{~b}$ & $\mathrm{Nt}^{*}$ & & $\mathrm{n}^{\underline{0}}$ & $\%$ \\
\hline Pernambuco & 4 & 1 & & & & 2 & 8 & & & & & 15 & 5,9 \\
\hline Bahia & 2 & & & & & & & & & & & 2 & 0,8 \\
\hline Goiás & 1 & & & & & & 3 & 1 & & 1 & 4 & 10 & 4,0 \\
\hline Distrito Federal & 2 & & & & & & 2 & & & & & 4 & 1,6 \\
\hline Minas Gerais & & & & & & & 3 & & & & & 3 & 1,2 \\
\hline Rio de Janeiro & 24 & 4 & & 1 & 2 & 1 & 45 & 3 & 1 & & & 81 & 31,7 \\
\hline São Paulo & 28 & & 1 & & & 4 & 87 & & & & & 120 & 47,0 \\
\hline Paraná & 11 & 1 & & & & & 4 & & & & & 16 & 6,2 \\
\hline Rio Grande do Sul & 2 & & & & & & 2 & & & & & 4 & 1,6 \\
\hline Total $\mathrm{n}^{\mathrm{0}}$ & 74 & 6 & 1 & 1 & 2 & 7 & 154 & 4 & 1 & 1 & 4 & 255 & 100,0 \\
\hline$\%$ & 29 & 2,4 & 0,4 & 0,4 & 0,8 & 2,7 & 60,3 & 1,6 & 0,4 & 0,4 & 1,6 & & \\
\hline
\end{tabular}

*Não tipável

Tabela 4 - Distribuição das espécies e sorovares de Listeria segundo as fontes de isolamento.

\begin{tabular}{|c|c|c|c|c|c|c|c|c|c|c|c|c|c|}
\hline \multirow[t]{2}{*}{ Fontes } & \multicolumn{7}{|c|}{ Listeria monocytogenes } & \multicolumn{3}{|c|}{ Listeria innocua } & \multirow[b]{2}{*}{ Listeria grayi } & \multicolumn{2}{|c|}{ Total } \\
\hline & $1 / 2 a$ & $1 / 2 \mathrm{~b}$ & $1 / 2 \mathrm{C}$ & $3 a$ & $4 a$ & $4 a b$ & $4 \mathrm{~b}$ & $6 a$ & $6 \mathrm{~b}$ & $\mathrm{Nt}^{*}$ & & $\mathrm{n}^{0}$ & $\%$ \\
\hline LCR & 36 & 1 & & & & 1 & 102 & & & & & 140 & 54,9 \\
\hline Sangue & 29 & 3 & 1 & 1 & 1 & 3 & 29 & & & & & 67 & 26,2 \\
\hline Fezes & 5 & 2 & & & 1 & & 11 & 3 & 1 & 1 & 4 & 28 & 11,0 \\
\hline Sangue*** & 2 & & & & & 1 & 7 & & & & & 10 & 4,0 \\
\hline Secreção vaginal & 2 & & & & & 2 & 2 & 1 & & & & 7 & 2,7 \\
\hline Adenite & & & & & & & 1 & & & & & 1 & 0,4 \\
\hline Fígado & & & & & & & 1 & & & & & 1 & 0,4 \\
\hline Lesão cutânea & & & & & & & 1 & & & & & 1 & 0,4 \\
\hline Total & 74 & 6 & 1 & 1 & 2 & 7 & 154 & 4 & 1 & 1 & 4 & 255 & 100,0 \\
\hline
\end{tabular}

*Não tipável. **Transplantados renais

incluindo dos transplantados renais, se tem um equilíbrio entre os sorovares $4 \mathrm{~b}$ e $1 / 2$ a, mas tendo uma diferença significativa para o primeiro tipo $(\mathrm{p}=0,004)$. Ao se comparar com o total de isolamentos, a diferença da distribuição entre as amostras isoladas de líquor e sangue é estatisticamente significante $(\mathrm{p}=0,04)$.

Todavia, apenas do sangue foi possível identificar todos os sete sorovares de L. monocytogenes caracterizados.

Na distribuição gráfica das frequiências das listérias, mas com ênfase nos sorovares mais incidentes nos três decênios (Figura 1), ficou evidente a predominância do sorovar $4 \mathrm{~b}$ em todas as fases, em particular no período de 1969-1978, que estatisticamente foi mais significante em confronto com os demais decênios $(\mathrm{p}=0,02)$. É interessante que na etapa de 1980-1989, a

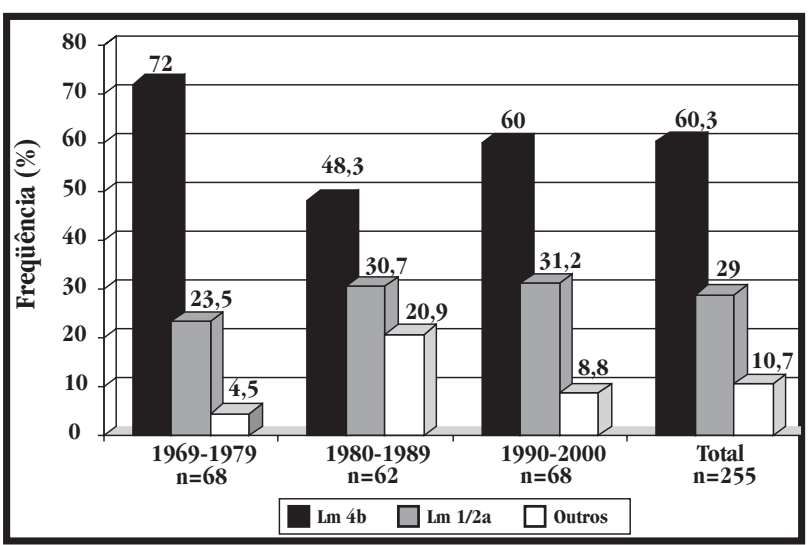

Figura 1 - Freqüência dos sorovares prevalentes de Listeria monocytogenes (1/2a e 4b) em relação aos demais sorotipos (1/2b, 1/2c, 3a, 4a, 4ab) e as espécies Listeria innocua e Listeria grayi. 
relação entre os sorotipos $4 \mathrm{~b} \mathrm{e} 1 / 2$ a, foi mais estreita, como resultado da maior recepção de cepas dos estados do Paraná e Rio de Janeiro, pertencentes ao último sorovar.

\section{DISCUSSÃO}

A listeriose é relativamente rara em crianças e adultos imunocompetentes, razão pela qual apresenta discreta incidência na maior parte do mundo. Talvez em decorrência desse fato, poucas foram as áreas que se detiveram em estudar o problema, citando-se como modelo as análises realizadas nos Estados Unidos da América em 1986, envolvendo cinco regiões, que indicaram uma incidência anual de 0,7 casos por $100 \mathrm{mil}$ habitantes. A taxa mais elevada se concentrou na listeriose perinatal com 12,7 casos por 100 mil nascidos vivos, embora em Los Angeles foi significativamente mais alta, com 23,7/100 $\mathrm{mil}^{32}$. Com as medidas preventivas de educação sanitária adotadas no período de 1989-1993, houve uma acentuada queda da incidência da listeriose, segundo Tappero cols ${ }^{35}$.

$\mathrm{Na}$ Europa, representada pela Grã-Bretanha, França e Alemanha, os cálculos revelaram a ocorrência de 2 a 3 casos por milhão de habitantes, de acordo com as citações de McLauchlin ${ }^{21}$ e Buchanan cols ${ }^{3}$. Não obstante 0 total desconhecimento da incidência da listeriose humana em nosso meio é admissível supor que nas regiões sul e sudeste, onde a frequiência de isolamentos de $L$. monocytogenes de casos clínicos tem sido mais comum ${ }^{11} 12$ a taxa seja similar as situações supramencionadas.

Já nas populações de maior risco, imunocomprometidos e que podem atingir até $20 \%$ da população total de um país desenvolvido $^{3}$ e que estão representados pelas gestantes, neonatos, transplantados renais, pacientes aidéticos, HIV infectados e com neoplasias, além de uma gama imensa de doenças crônico-degenerativas envolvendo principalmente idosos, as incidências variaram entre 200 casos por $100 \mathrm{mil}$ pacientes transplantados renais ${ }^{26}$ a 52 e 115/100 mil pacientes HIV infectados e com AIDS, respectivamente ${ }^{16}$.

Uma das dificuldades encontradas na presente investigação residiu na caracterização de caso de listeriose, em vista das deficiências dos dados clínicos e demográficos recebidos pelo laboratório. A solução encontrada para o problema, foi adotar o critério de McLauchlin ${ }^{20}$, que considerou como caso de doença quando a $L$. monocytogenes era isolada de um sítio anatômico normalmente estéril. Dessa forma, $220(86,3 \%)$ casos foram enquadrados como doentes e 35 (13,7\%) de indivíduos aparentemente normais, cujos isolamentos se originaram de fezes (28) e de secreção vaginal e/ou cervical (7), mesmo quando havia a suspeita clínica de abortos habituais, que propiciaram o isolamento de $L$. monocytogenes sorovares $1{ }_{2}$ a (2) e 4b (2) em quatro pacientes do Rio de Janeiro.

Outro ponto a ser abordado, situa-se nas definições de casos perinatal e não perinatal, caracterizados por Rocourt cols ${ }^{28}$, como o isolamento concomitante de $L$. monocytogenes de sítios anatômicos estéreis da parturiente e do neonato ou feto na primeira condição e quando o isolamento bacteriano fica restrito ao organismo materno ou do neonato de áreas estéreis, caracteriza a infecção não perinatal. Salienta-se que nesta pesquisa, a infecção perinatal foi reconhecida em três casos oriundos do Paraná, São Paulo e Rio de Janeiro através de hemocultivos e sendo identificados os sorovares $1 / 2$ a do primeiro local e 4b, dos demais. No terreno da hipótese, admite-se que 0 pequeno número de casos relatados, decorra da definição bastante específica, mas pouco sensível sob o prisma clínico, associado a precariedade ou ausência de informações que acompanhavam o espécime clínico e/ou a amostra isolada.

A mesma dificuldade também se tem para a caracterização de casos esporádicos de listeriose como nosocomiais. Os conceitos sobre casos hospitalares são díspares quanto aos limites de um período do acontecimento. Alguns são definidos como àqueles em que o isolamento de L. monocytogenes ocorreu após três dias de admissão do paciente ${ }^{14}$, enquanto outros ampliam o prazo entre 10 a 51 dias pós-internação ${ }^{24}$. Adotandose a primeira orientação, registram-se cinco casos como listeriose nosocomial e não perinatal na presente amostragem. 0 problema envolveu quatro recém-natos em hospital de São Bernardo do Campo, São Paulo, em janeiro de 1997, com os isolamentos do sorovar $4 \mathrm{~b}$ dos líquidos cefalorraquidianos.

É evidente na amostragem a predominância de isolados de líquidos cefalorraquidianos em relação aos de sangue em todos os grupos etários (Tabela 1). Aliás, este acontecimento foi descrito por McLauchlin ${ }^{2022}$ e Büla cols ${ }^{4}$, quando afirmaram que as localizações de L. monocytogenes no sistema nervoso central foram mais comuns em $65 \%$ dos indivíduos não portadores de algum fator de risco, particularmente o estado de imunossupressão. Em contraposição, nos pacientes com infecções não perinatais com fatores predisponentes, a bacteriemia é mais frequiente entre 67 a 93\% dos casos. No entanto, é fundamental levar em conta que a proporção de pacientes previamente saudáveis antes ou durante a infecção pela Listeria ou qualquer outra etiologia microbiana permanece na maioria das vezes como obscura. Sem dúvida que o termo sadio deve ser usado com cautela, uma vez que o estado imunológico dos pacientes não é rotineiramente investigado e uma possível imunodepressão transitória excepcionalmente será detectada ${ }^{7}$. Tendo em vista as considerações mencionadas, provavelmente nas amostras analisadas, apenas aquelas provenientes dos dez pacientes transplantados renais, com hemocultura positiva e sem comprometimento do sistema nervoso central ${ }^{8}$, isto é, com bacteriemia primária se compatibilizaram com as observações supramencionadas ${ }^{4022}$.

$\mathrm{Na}$ distribuição geográfica dos isolados (Tabela 2 e 3), houve uma predominância de $L$. monocytogenes nas regiões sudeste e sul do país $(87,8 \%)$, embora tenha sido também detectada em estados do centro-oeste e nordeste (10,9\%). Para alguns autores ${ }^{15} 27$ a listeriose compromete mais as populações de países ou de regiões industrializadas em confronto com os baixos índices relacionados nas áreas em desenvolvimento. Enfatizam ainda que, mesmo em regiões desenvolvidas, existe uma diferença da incidência de listeriose nas classes sociais, que via de regra aquelas mais elevadas foram as mais comprometidas. 
0 problema da maior prevalência de listeriose nas populações socioeconômicas mais privilegiadas, incluindo um sistema de saúde mais avançado, tem uma estreita conotação com a alimentação, uma vez que $32 \%$ dos casos esporádicos podem ser atribuídos ao consumo de alimentos contaminados com $L$. monocytogenes ${ }^{32}$. Na atualidade o problema tende a se agravar com o incremento do hábito da população consumir os alimentos prontos ${ }^{5}$, cujos ingredientes, por vezes são estocados à temperatura de refrigeração por um certo período, mas que favorece a multiplicação da Listeria ${ }^{25}$.

É oportuno ressaltar que, praticamente de todas as regiões do Brasil, foi detectada L. monocytogenes em diversos alimentos de origens animal e vegetal ${ }^{10}$, caracterizando sua ampla disseminação em natureza. Logicamente que, a propagação ao homem ficará na dependência de propriedades inerentes do agente, como a dose infectante de uma raça virulenta e acima de tudo de uma população consumidora de alimentos prontos e cada vez mais portadora de fatores de risco predisponentes. Por sinal, este problema foi muito bem analisado por Buchanan cols $^{3}$, que desenvolveram um modelo matemático relacionando os parâmetros mencionados, com ênfase no consumo de certos alimentos, visando o desenvolvimento de uma fórmula capaz de calcular a incidência da listeriose.

Além dos aspectos discutidos, salienta-se a figura de portadores fecais, descrita em várias oportunidades e em diferentes populações ${ }^{191317181931}$. Tais pesquisas demonstraram que a frequiência de indivíduos assintomáticos normais ou pósinfecção listeriótica foi extremamente variável e funcionando na maioria das vezes como portadores temporários. Transportando-se o problema para a presente investigação, observa-se na Tabela 1, a ocorrência de representantes do gênero Listeria nas fezes em quase todos os grupos etários, predominando nas faixas de 31 a 60 anos 22 (78,5\%) portadores. Na caracterização de sorovares de $L$. monocytogenes (Tabela 4), prevaleceram os tipos $4 \mathrm{~b}$ (11 amostras) e $1 \frac{1}{2}$ a (5), compatibilizando-se com a maioria das citações. Registra-se ainda a ocorrência das espécies não patogênicas, L. innocua (5) e L. grayi (4). Curiosamente, raras são as citações na literatura sobre a presença da última espécie em material clínico humano ${ }^{2328}$ e a veiculação dessas espécies não patogênicas certamente, se faz através dos alimentos, considerando que a $L$. innocua sorovar 6 a predominou nos produtos de laticínios e cárneos (processados e não processados) superando L. monocytogenes sorovar $1 / 2 \mathrm{a}$ em derivados do leite e $4 \mathrm{~b} \mathrm{e} 1 / 2 \mathrm{~b}$ em carnes e embutidos ${ }^{10}$. Diante da situação retratada, corrobora-se com a hipótese da infecção endógena, isto é, resultante da ingestão de alimentos contaminados e da colonização da bactéria no trato entérico, particularmente em pacientes sob a intervenção de fármacos imunossupressores, que além de diminuir a resistência do indivíduo à infecção, danificam também a mucosa $a^{34}$.

Um acontecimento interessante refere-se a heterogeneidade de sorovares reconhecidos nas hemoculturas em relação àqueles de líquor (Tabela 4). É possível advogar que a distribuição de diversos sorovares, ou mesmo de um sorotipo, reflete e depende essencialmente, da presença dos fatores de virulência ${ }^{2}$, da capacidade de sobrevivência no meio ambiente, incluindo os alimentos $\mathrm{s}^{653032}$ e da imunocompetência da fonte de infecção ${ }^{1212734}$. Tais premissas se coadunam com o raciocínio daqueles que consideram que todos os sorovares de L. monocytogenes são potencialmente patogênicos para o homem e animais ${ }^{202228}$, indiferente das características feno e genotípicas ${ }^{2}$.

Em síntese, a ocorrência de L. monocytogenes na espécie humana não é um fato inusitado para o nosso meio, inclusive reproduzindo resultados similares aos de outras partes do mundo quanto à prevalência de sorovares. Por outro lado, a investigação teve como intento básico alertar sobre a existência do problema, em nosso meio, tendo em vista 0 discreto conhecimento dos diversos ramos profissionais sobre os aspectos clínico-epidemiológicos e laboratoriais da Listeria monocytogenes e da listeriose.

\section{AGRADECIMENTOS}

A todos os colegas e instituições que colaboraram com a remessa de amostras. Aos técnicos Rosemary Ribeiro, Deise Paranhos Feitosa (in memoriam), Junair Ribeiro e Darcília Maria de Andrade, do Departamento de Bacteriologia do Instituto Oswaldo Cruz (FIOCRUZ), pelo apoio ao longo do trabalho.

\section{REFERÊNCIAS BIBLIOGRÁFICAS}

1. Bojsen-Møller J. Human listeriosis: diagnostic, epidemiologic and clinical studies. Acta Pathologica Microbiologica Scandinavica [B] 229 (suppl): 72-92, 1972.

2. Brosch R, Catimel B, Milon G, Buchrieser C, Vindel E, Rocourt J. Virulence heterogeneity of Listeria monocytogenes strains from various sources (food, human, animal) in immunocompetent mice and its association with typing characteristics. Journal of Food Protection 56: 296-301, 1993.

3. Buchanan RL, Damert WG, Whiting RC, Van Schothorst M. Use of epidemiologic and food survey data to estimate a purposefully conservative dose-response relationship of Listeria monocytogenes levels and incidence of listeriosis. Journal of Food Protection 60: 918-922, 1997.

4. Bülla CJ, Bille J, Glauser MP. An epidemic of food-borne listeriosis in western Switzerland: description of 57 cases involving adults. Clinical Infectious Diseases 20: 66-72, 1995.

5. Farber JM, Harwig J. The Canadian position on Listeria monocytogenes in ready-to-eat foods. Food Control 7: 253-258, 1996.

6. Farber JM, Peterkin PI. Listeria monocytogenes, a food-borne pathogen. Microbiological Reviews 55: 476-511, 1995.

7. Goday A, Lozano F, Santamari J, Gallard T, Tolasa E. Transient immunologic defect in a case of Listeria rhombencephalitis. Archives of Neurology 44: 666-667, 1987

8. Hofer CB, Melles CEA, Hofer E. Listeria monocytogenes in renal transplant recipients. Revista do Instituto de Medicina Tropical de São Paulo 41: 375 $377,1999$.

9. Hofer E. Pesquisa sobre a ocorrência de Listeria monocytogenes em fezes humanas. Revista da Sociedade Brasileira de Medicina Tropical 3: 109-116, 1974.

10. Hofer E. Três decênios de experiência sobre Listeria no Brasil. In: Mercadante AZ, Bobbio FO, Bobbio PA, Pereira JL, Pastore GM (eds) Ciência de Alimentos-avanços e perspectivas, Faculdade de Engenharia de Alimentos, Universidade Estadual de Campinas, Campinas, vol. II, p.111-115, 2001

11. Hofer E, Pessoa GVA, Melles CEA. Listeriose humana. Prevalência dos sorotipos de Listeria monocytogenes isolados no Brasil. Revista do Instituto Adolfo Lutz 44: 125-131, 1984. 
12. Hofer E, Ribeiro R, Feitosa DP. Species and serovars of the genus Listeria isolated from different sources in Brazil from 1971 to 1997. Memórias do Instituto Oswaldo Cruz 95: 615-620, 2000.

13. Jensen A. Excretion of Listeria monocytogenes in faeces after listeriosis: rate, quantity and duration. Medical Microbiology Letters 2: 176-182, 1983.

14. Jensen A, Frederiksen W, Gerner-Smidt P. Risk factors for listeriosis in Denmark, 1989-1990. Scandinavian Journal of Infectious Diseases 26: 171$178,1994$.

15. Jones EM, McCulloch SY, Reeves DS, Macgowan AP. A 10 year survey of the epidemiology and clinical aspects of listeriosis in a provincial English city. Journal of Infection 29: 91-103, 1994.

16. Jurado RL, Farley MM, Pereira E, Harvey RC, Schuchat A, Wenger JD, Stephens DS. Increased risk of meningitis and bacteriemia due to Listeria monocytogenes in patients with human immunodeficiency virus infection. Clinical Infectious Diseases 17: 224-227, 1993.

17. Kampelmacher EH, Van Noorle Jansen LM. Isolation of Listeria monocytogenes from feces of clinically healthy humans and animals. Zentralblatt für Bakteriologie und Hygiene Abt I 221: 353-359, 1969.

18. Macgowan AP, Marshall RJ, Mackay IM, Reeves DS. Listeria faecal carriage by renal transplant recipients, haemodialysis patients and patients in general pratice: its relation to season, drug therapy, foreign travel animal exposure and diet. Epidemiology and Infection 106: 157-166, 1991.

19. Mascola L, Sorvillo F, Goulet V, Hall B, Weaver R, Linnan M. Fecal carriage of Listeria monocytogenes-Observations during a community-wide, common-source outbreak. Clinical Infectious Diseases 15: 557-558, 1992.

20. McLauchlin J. Distribution of serovars of Listeria monocytogenes isolated from different categories of patients with listeriosis. European Journal of Clinical Microbiology and Infectious Diseases 9: 210-213, 1990.

21. McLauchlin J. The relationship between Listeria and listeriosis. Food Control 7: 187-193, 1996.

22. McLauchlin J. The pathogenicity of Listeria monocytogenes: a public health perspective. Reviews in Medical Microbiology 8: 1-14, 1997.

23. Nojimoto ITI, Souza SR, Valadão LM. Ocorrência de Listeria spp em crianças da Cidade de Goiânia-Goiás. Revista Brasileira de Análises Clínicas 29: 73-74, 1997.
24. Nolla-Sallas J, Antó JM, Almela M, Coll P, Gasser I, Plasencia A, Collaborative Study group of Listeriosis of Barcelona. Incidence of Listeriosis in Barcelona, Spain, in 1990. European Journal of Clinical Microbiology and Infectious Diseases 12: 157-161, 1993.

25. Pinner RW, Schuchat A, Swaminathan B, Hayes PS, Deaver KA, Weaver RE, Plikaytis BD, Reeves M, Broome CV, Wenger JD, Listeric Study Group. Role of food in sporadic listeriosis II. Microbiologic and epidemiologic investigation. Journal of the American Medical Association 267: 2046-2050, 1992.

26. Rebière I, Goulet V. La Listériose: revue générale et référence à l'épidémie française de 1992. Lettre de L'Infectiologue 8: 130-135, 1993.

27. Rocourt J. Risk factors for listeriosis. Food Control 7: 195-202, 1996.

28. Rocourt J, Jacquet Ch, Bille J. Human listeriosis 1991-1992. WHO/FNU/ FOS/97.1. World Health Organization, Geneva, 1997.

29. Rocourt J, Schrettenbrunner A, Seeliger HPR. Différenciation biochimique des groups génomiques de Listeria monocytogenes (sensu lato). Annales de L'Institute Pasteur Microbiologie 134A: 65-71, 1983.

30. Rocourt J, Seeliger HPR. Distribution des espèces du genre Listeria. Zentralblatt für Bakteriologie und Hygiene A259: 317-330, 1985.

31. Schuchat A, Deaver KA, Hayes PS, Graves L, Mascola L, Wenger JD. Gastrointestinal carriage of Listeria monocytogenes in household contacts of patients with listeriosis. The Journal of Infectious Diseases 167: 1261-1262, 1993.

32. Schuchat A, Deaver KA, Wenger JD, Plikaytis BD, Mascola L, Pinner RW, Reingold AL, Broome CV, Listeria Study Group. Role of foods in sporadic listeriosis. I. Case-control study of dietary risk factors. Journal of the American Medical Association 267: 2041-2045, 1992.

33. Seeliger HPR, Höhne K. Serotyping of Listeria monocytogenes and related species. In: Bergan T, Norris JR (ed) Methods in Microbiology, Academic Press, London, vol 13, p. 31-49, 1979.

34. Skogberg K, Syrjänen NJ, Jahkola M, Renkonen O-V, Pavoneen J, Ahonen J, Kontiainen S, Ruutu P, Valtonen V. Clinical presentation and outcome of listeriosis in patients with and without immunosuppressive therapy. Clinical Infectious Diseases 14: 815-821, 1992.

35. Tappero JW, Schuchat A, Deaver KA, Mascola L, Wenger JD. Reduction in the incidence of human listeriosis in the United States. Effectiveness of prevention efforts? Journal of the American Medical Association 273: 1118-1122, 1995. 